\title{
When objects are close to me: Affordances in the peripersonal space
}

\author{
Marcello Costantini • Ettore Ambrosini · \\ Claudia Scorolli • Anna M. Borghi
}

Published online: 5 February 2011

(C) Psychonomic Society, Inc. 2011

\begin{abstract}
In the present study, we investigated, using language, which motor information is automatically activated by observing 3-D objects (i.e., manipulation vs. function) and whether this information is modulated by the objects' location in space. Participants were shown 3-D pictures of objects located in peripersonal versus extrapersonal space. Immediately after, they were presented with function, manipulation, or observation verbs (e.g., "to drink," "to grasp," "to look at") and were required to judge whether the verb was compatible with the presented object. We found that participants were slower with observation verbs than with manipulation and function verbs. With both function and manipulation verbs, participants were faster when objects were presented in reachable space. Interestingly, the fastest response times were recorded when
\end{abstract}

M. Costantini $(\varangle) \cdot$ E. Ambrosini

Department of Neuroscience and Imaging,

University G. d'Annunzio,

Via dei Vestini, 33,

66013 Chieti, Italy

e-mail: marcello.costantini@unich.it

C. Scorolli · A. M. Borghi

University of Bologna,

Bologna, Italy

A. M. Borghi

National Research Council (CNR),

Rome, Italy

\author{
A. M. Borghi $(\bowtie)$ \\ Department of Psychology, \\ Viale Berti Pichat, 5, \\ 40100 Bologna, Italy \\ e-mail: annamaria.borghi@unibo.it
}

participants read function verbs while objects were presented in the accessible space. Results suggest that artifacts are first conceived in terms of affordances linked to manipulation and use, and that affordances are differently activated, depending on context.

Keywords Affordance $\cdot$ Manipulation $\cdot$ Function . Peripersonal space $\cdot$ Tool

Gibson (1979) defined affordances as properties in the environment that are relevant for an organism's goals. Recently, Ellis and Tucker (2000) proposed to adopt the term microaffordance. Microaffordances differ from Gibsonian affordances, since they typically refer to simple and specific kinds of interactions with objects, such as reaching and grasping. As compared with Gibson's view, recent literature on affordances emphasizes the presence of brain assemblies that represent objects and relations with objects. On the behavioral side, studies on compatibility effects showed that observing pictures of objects or real objects potentiates specific motor acts - that is, the common reaching and grasping actions we typically perform with them (Tucker \& Ellis, 1998, 2001). For example, observing a handled cup leads to the activation of the movements aimed at reaching for its handle, and the grip adequate to grasp it in order to drink from it (Tucker \& Ellis, 1998, 2001). These results reveal that manipulable objects are represented in terms of actions that can be realistically executed. The category of artifacts, and particularly of tools (e.g., a nutcracker), can be somewhat peculiar (Creem-Regehr \& Lee, 2005). Behavioral evidence has demonstrated that, in categorization tasks, artifacts are responded to more slowly than natural objects, probably due to the fact that they activate manipulation as well as functional information (e.g., putting the hammer in the 
toolbox vs. hammering a nail [Anelli, Nicoletti, \& Borghi, 2010; Gerlach, 2009]). As far as neural activation is concerned, neurophysiological evidence showed that the simple observation of objects leads to the activation of the canonical neuron system (Murata et al., 1997), and brain imaging studies have shown that, although natural objects activate occipital areas, tools are represented in the ventral premotor cortex (for a review, see Martin, 2007).

Thus, behavioral, neurophysiological, and brain imaging studies have demonstrated that seeing objects activates motor responses. This evidence leaves an issue unanswered: Does the object evoke a compatible action regardless of the possibility to directly act on it? In the present work, we aimed to investigate this issue by presenting pictures of artifacts in operational (peripersonal and reachable by a simple arm movement) and in nonoperational (extrapersonal and nonreachable) space. In addition, we intended to verify whether the kind of action elicited by objects (i.e., manipulation vs. function) is modulated by their location in operational versus nonoperational space. Following Bub, Masson, and Cree (2008), we refer to manipulation as all the grasping gestures accomplished to pick up an object (volumetric/manipulation), whereas we define function as all the grasping gestures associated with using an object for its intended purpose (Pellicano et al., 2010). Consider a knife: We might use either a specific hand posture and grip in order to cut some bread with it (functional gesture) or a different grip in order to put it into a drawer (volumetric/manipulation gesture).

Current results are rather conflicting as to whether information related to manipulation and function are automatically activated (e.g., Boronat et al., 2005). For example, Kellenbach, Brett, and Patterson (2003) found that no regions of the cortex were more activated by function relative to action judgments in relation to artifacts. Creem and Proffitt (2001) used a dual-task paradigm and found that function information must be activated to perform appropriate actions with objects, such as grasping a handle in an appropriate way.

In the present study, we addressed whether artifacts evoke action information differently depending on whether they are located within or outside the peripersonal space. A powerful way to study the way objects are represented is to use verbal labels. In our study, participants were presented with verbs referring to function, manipulation, and observation (e.g., "to drink," "to grasp," "to look at") and were required to judge whether the verb they read was compatible with the previously presented object, which worked as a prime. Hence, we used response times (RTs) to linguistic stimuli in order to understand which kind of information is activated while observing artifacts. Specifically, we focused on whether and how the presentation of an object in the reachable versus nonreachable space (peri- vs. extra-personal space) influences the way we represent it. This paradigm allows us to make the two following predictions:

1. If activation of potential action with objects is modulated by the potentiality to interact with it, then manipulation and function verbs should be responded to more quickly when objects are in the peripersonal space. Conversely, we do not expect any difference in responding to observation verbs for objects presented in the peripersonal and extrapersonal space, given that observation does not require a physical interaction with the object.

2. If observation of artifacts evokes both manipulation and functional information, then manipulation and function verbs should be responded to more quickly than observation verbs.

\section{Method}

Participants

A total of 32 healthy subjects (17 males, mean age 33.5 years) took part in the experiment. All participants were native Italian speakers, had normal or corrected-tonormal visual acuity, and were right-handed according to self-report. They were naive as to the purpose of the experiment and gave their informed consent.

\section{Materials}

We selected 12 critical manipulation-verb/function-verb/ object triples from a sample of 30 triple groupings. In order to perform the selection, we asked 48 Italian participants (22 males, mean age 30.9 years) to judge how compatible each verb was with each object. They were required to provide ratings on a $0-100$ visual-analogical scale (Not compatible-Very compatible) by making a cross on a line. We selected the triples (manipulation verb/function verb/ object) with the highest compatibility scores. That is, for each object, we had a highly compatible manipulation and function verb. As far as the observation verbs are concerned, we used only four different verbs, due to the difficulty in finding a higher number of different verbs.

The experimental stimuli were images and verbs. Images consisted of red/cyan anaglyph stereo pictures depicting a 3-D room displaying a table with an object placed on top of it. Twelve common objects were used (see the Appendix ). All of the objects used would normally be grasped with a power grip and were presented with the handle or the graspable part toward the right. Images were created by 
means of 3-D Studio Max ${ }^{\mathrm{TM}}$ and StereoPhoto Maker. Using $\mathrm{red} /$ cyan anaglyph stereo pictures allowed us to present the objects either within the peripersonal $(50 \mathrm{~cm})$ or extrapersonal $(170 \mathrm{~cm})$ space of participants (See Fig. 1a). Verb stimuli consisted of three lists of Italian verbs in the imperative form. The three lists referred to function, manipulation, and observation verbs (see the Appendix). Each verb was matched with only one object, with the exception of the observation verbs.

\section{Appendix mentioned}

Procedure

Participants sat in front of a computer screen at a distance of approximately $57 \mathrm{~cm}$, wearing anaglyph 3-D glasses. Each trial consisted of the presentation of an object for $500 \mathrm{~ms}$ and was followed - after a delay of 50 or $100 \mathrm{~ms}$ by a verb presented at the center of the screen that lasted 1,500 ms (see Fig. 1b). Each trial began with the participant resting the right index finger on a response button. Participants were instructed to respond if the object-verb combination was appropriate, and to refrain from responding if the object-verb combination did not make sense (catch trials). Catch trials were created by combining objects with verbs related to other objects (e.g., object/ verb: ball/“to plug up;" ball/“to drink"). Responses were made by lifting the finger from the response button and then making an unspecified grasping movement toward the computer screen. During the intertrial interval, a white fixation cross was presented for 1,000 ms.
The presentation of the stimuli and the recording of the participants' responses were controlled by a custom software (Galati et al., 2008) and were implemented in MATLAB using Cogent 2000 (developed at FIL and ICN, UCL, London, UK) and Cogent Graphics (developed by John Romaya at the UCL, London, UK).

For every object, all of the three types of verbs were presented twice in both peripersonal and extrapersonal space; therefore, there were 24 trials per condition for a total of 144 trials plus 48 catch trials (25\%), lasting approximately $10 \mathrm{~min}$. At the end of the experiment, participants were requested to estimate the distance of the objects in relation to their bodies. The stimuli presented in the peripersonal and extrapersonal spaces were judged as being at a distance of $50 \pm 14 \mathrm{~cm}$ and $190 \pm 42 \mathrm{~cm}$ from the participants.

\section{Results}

Trials in which participants failed to respond (9.1\%) were excluded from the analysis on RTs. The mean RTs were calculated for each condition; responses longer than two standard deviations from the individual mean were treated as outliers (4.6\%). Data were entered in a two-way ANOVA with location of the object (peripersonal vs. extrapersonal space) and verb (function vs. manipulation vs. observation) as within-subjects factors.

An RT analysis revealed a significant main effect of object location, $F(1,31)=19.8 ; p<.001$, with higher RTs on extrapersonal trials $(M=798 \mathrm{~ms})$ than on peripersonal trials $(M=770 \mathrm{~ms})$.
Fig. 1 a Example of experimental stimuli. Red/cyan anaglyph stereo pictures were used, allowing the presentation of the objects either within the peripersonal $(50 \mathrm{~cm})$ or extrapersonal $(170 \mathrm{~cm})$ space. $\mathbf{b}$

Experimental timing

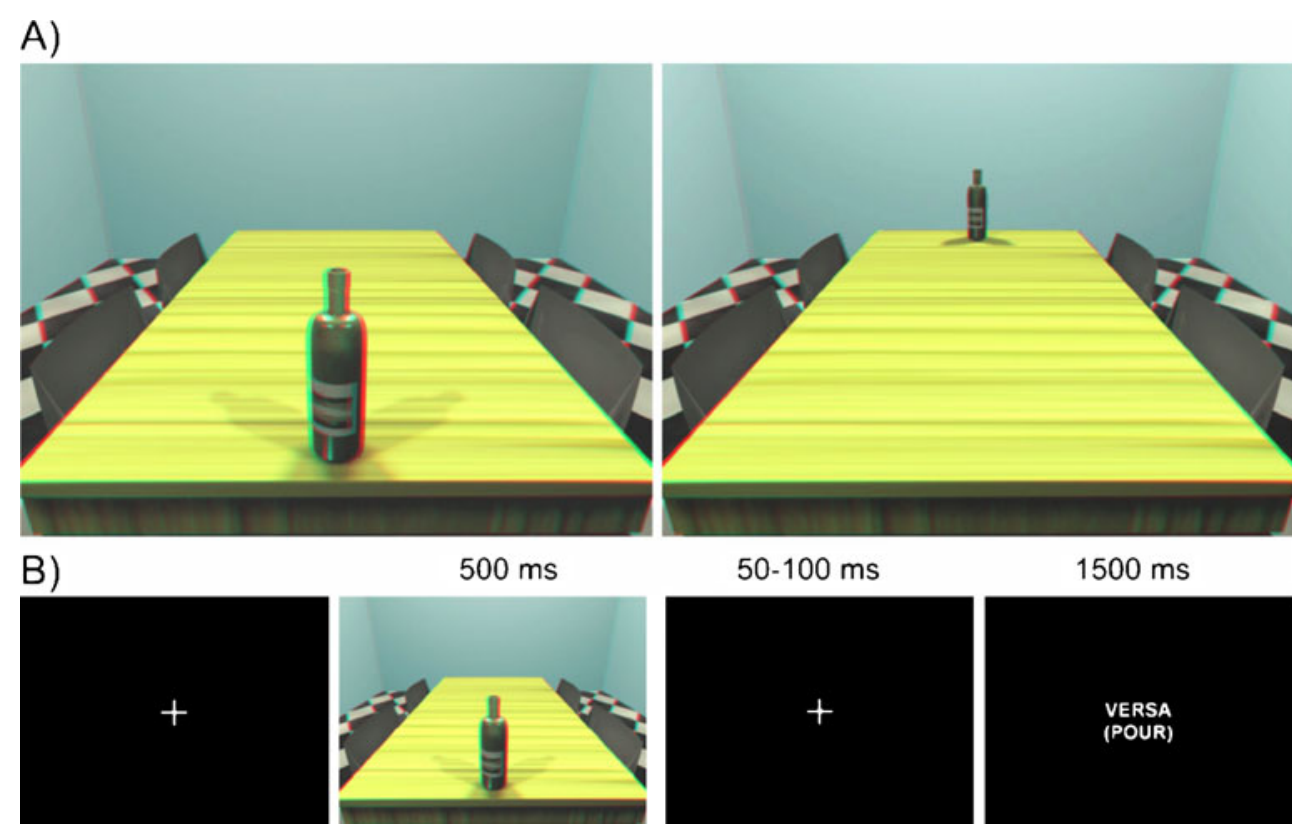


The main effect of verb was also significant, $F(1,31)=$ 24.9; $p<.001$ ). A post hoc analysis (Newman-Keuls) revealed RTs to function trials $(M=737 \mathrm{~ms})$ to be faster than both RTs to manipulation $(M=792 \mathrm{~ms})$ and observation $(M=823 \mathrm{~ms})$ trials, which in turn did differ from each other. It is important to note here that the main effect of verb is unlikely to be due to differences in the frequency of use. Indeed, we checked for it (DeMauro, Mancini, Vedovelli, \& Voghera, 1993), and we found the following word frequencies: function $=20$; manipulation $=$ 19; observation $=98$. Thus, although observation verbs had the highest frequency of use, they had the slowest RTs.

Crucially, an RTs analysis revealed a significant Location $\mathrm{x}$ Verb interaction, $F(2,62)=7.4 ; p<.01$; Fig. 2). A Newman-Keuls post hoc analysis showed that although RTs to observation verbs were comparable in the peripersonal and extrapersonal space (mean RTs: 822 vs. $823 \mathrm{~ms}$ ), they were faster on peripersonal than on extrapersonal space for both function (mean RTs: 711 vs. $763 \mathrm{~ms}$ ) and manipulation (mean RTs: 775 vs. 809 ms) verbs. Moreover, within the peripersonal space, RTs to function verbs were faster than RTs to manipulation verbs $(p<.01)$. Finally, RTs to function verbs in the extrapersonal space were faster than RTs to observation verbs in the same space.

A similar ANOVA was carried out on the number of errors. A significant main effect of verb was observed, $F(2,62)=$ $3.82 ; p<.05)$. A post hoc analysis showed that more errors occurred in response to observation $(M=2.75)$ as compared with function $(M=1.77)$ and manipulation $(M=1.97)$ verbs, which in turn did not differ from each other.

An ANOVA also revealed a significant Location $\mathrm{x}$ Verb interaction, $F(2,62)=5.7 ; p<.01$. A Newman-Keuls post hoc analysis showed that more errors occurred with observation verbs $(M=2.94)$ in the peripersonal space as compared with manipulation $(M=2.06)$ and function $(M=$

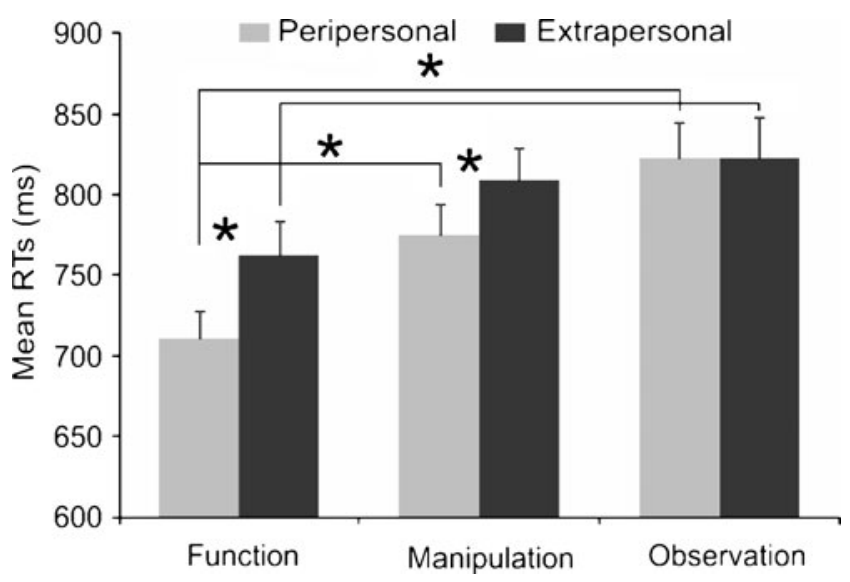

Fig. 2 Mean reaction times (RTs) in the experimental conditions. Error bars indicate standard errors
1.31) verbs, which in turn differed from each other $(p<.05$ in all cases). No other comparisons were significant.

\section{Discussion}

Our most important result clearly shows that the activation of the potential actions to perform with objects is modulated by the current context and by object accessibility. RTs for manipulation and function verbs differed depending on the object location in the peri- vs. extrapersonal space, whereas RTs for observation verbs did not differ depending on the distance of the object from the body. This suggests that objects are represented in a flexible way, and that motor information related to both manipulation and use of objects is more relevant when a physical interaction with an object is effectively possible.

This finding is in line with recent results by Costantini, Ambrosini, Tieri, Sinigaglia, and Committeri (2010), who investigated whether and to what extent the effective processing of affordances of an object might depend on its spatial location. Their results showed that the perception of affordance suggests a motor act only when the object is presented within the operational space of participants. Our results are novel, but they also strengthen and extend the results found by Costantini et al., since we used verbs to determine the role of observation and action in the emergence of affordances (Borghi, 2004; Borghi \& Riggio, 2009). Specifically, pictures of objects differentially primed verbs referring either to observation or action. Our study suggests that reading verbs activates a simulation of potential interactions with objects; therefore, our finding is in line with the view that language is grounded in the sensorimotor system (Glenberg \& Robertson, 2000; Scorolli, Borghi, \& Glenberg, 2009). This result has interesting theoretical implications for the literature on affordances. It warns that the claim that affordances are automatically activated should be viewed with caution, and it suggests that affordances are context-dependent relations (Chemero, 2003, 2009; Costantini et al., 2010; Costantini \& Sinigaglia 2011). Indeed, it reveals that action information is mostly activated when the possibility to effectively interact with an object exists.

Even if the interaction between the action/observation verbs and the peri- vs. extrapersonal space is by far the most important finding, a further result is worth mentioning - the fact that both function and manipulation verbs were processed faster than observation verbs. This is compatible with the idea that artifacts are represented in terms of the actions they elicit (Borghi, 2004). Previous findings suggest that visual observation of objects activates a motor simulation of the possible actions to perform with them (Gallese, 2009; Jeannerod 2007); our results extend this evidence, revealing 
that the motor simulation evoked while observing objects is spatially constrained.

Alternative explanations of the advantage of observation over manipulation and function verbs can be addressed. One could explain the difference on the basis of our design: Each observation verb was presented more frequently during the experiment than was each manipulation and function verb. Moreover, there were not catch trials with observation verbs, so they were always responded to. However, our results are in contrast with a frequencybased account: Indeed, observation verbs were responded to more slowly than were both manipulation and function verbs. Most importantly, consider that our task required participants to respond if the object-verb combination was appropriate (catch trials were only $25 \%$ ), and that we did not use different blocks for each kind of verb. Due to the mixed design we used, it would be improbable that participants formed separate categories for each verb kind (observation, function, and manipulation) and decided to respond to observation verbs, but not to the other verbs. To accomplish the task, it is much more probable that they simply responded to the sensibility of each combination.

An additional alternative explanation of our results can pertain to the specificity of the verb in each object-verb combination. One may argue that although observation verbs are rather unspecific because they can be combined with all objects, manipulation verbs are less specific, and function verbs are the most specific with regard to the selected objects. A closer examination of our results weakens this account. Indeed, RTs for observation verbs, regardless of the object location, did not significantly differ $(p=.25)$ from RTs to manipulation verbs referring to objects located in the extrapersonal space. Instead, they differed from RTs to manipulation verbs referring to objects located in the peripersonal space. The absence of a significant difference between observation verbs and manipulation verbs referring to objects in the far space strongly undermines the hypothesis that the difference between observation and manipulation verbs is due to their different degree of specificity with respect to the selected objects.

Now, let us turn to the difference between manipulation and function verbs. We interpret this difference as being compatible with the idea that seeing objects activates a motor prototype (Borghi \& Riggio, 2009; Menz, Blangaro, Kunze, \& Binkofski, 2010) that elicits a set of common actions. Our results suggest that this motor prototype includes the relevant affordances for object use (as the fastest responses obtained with function verbs indicate) and object manipulation.

We also considered alternative theoretical accounts of our results. A first possibility is that function verbs are responded to more quickly than are other verbs because they are more frequent. As described in the Method section, this explanation can be ruled out on the basis of an analysis of word frequencies, showing that observation verbs were more frequent than other verbs.

A further possibility is that the combination of objects with function verbs is simply easier than the combination of objects with manipulation verbs, given that the pattern of results holds for both the peri- and extrapersonal space. We consider two possibilities for this ease. One possibility is that it is easier due to the task at hand: For example, Jax and Buxbaum (2010) have shown that grasping an object according to its shape was slowed after interacting with the object functionally. However, the task we used simply required the participant to decide whether the object and the verb were compatible; it did not require one to judge pairs on the basis of their function, nor did it prime a specific interaction (manipulation/function) with the object. A further possibility is that functional verbs are easier because objects are consciously experienced more often in terms of their use than in terms of their manipulability. If this is the case, this would confirm our hypothesis. It should be noted here that faster responses to function rather than to manipulation verbs do not imply that only functional representations are activated: It is highly probable, as has been shown in recent studies (Bub et al., 2008; Jax \& Buxbaum, 2010), that both manipulation and function are activated, and that a competition between the two kinds of representation takes place.

The stronger activation of function over manipulation has strong implications concerning the neural basis of tool representation (Mahon, Schwarzbach, \& Caramazza, 2010). It provides a behavioral demonstration in support of the view according to which within the parietal cortex there is a distinction between two circuits: Posterior parietal cortex along the intraparietal sulcus is more devoted to manipulation (Binkofski et al., 1998; Menz et al., 2010), whereas the left inferior parietal lobule is linked to knowledge of tool use (Menz et al., 2010; Rizzolatti \& Matelli, 2003).

One further issue is worth mentioning. Note that we used 3-D images. We do not think that our results undermine findings on affordances obtained presenting 2-D images; however, they indicate that the operational space might represent an important factor worth considering.

Overall, we believe our results reveal, in a straightforward and simple way, both stable and flexible elements of the way in which we represent objects. When we observe artifacts, we activate the potential actions employed to perform with them. The advantage of manipulation and function verbs over observation verbs suggests that we activate both their function - that is, the most common actions we perform with them-and how to efficiently manipulate them. However, objects do not activate information in a stable and invariant way. Rather, knowledge on how to use and manipulate objects is most useful when objects are located close to us, in our peripersonal space. 
Author Note C.S. and A.M.B. were funded by the EU FP7 project "Emergence of communication in Robots through Sensorimotor and Social Interaction" (ROSSI), Grant Agreement 216125 (www.rossiproject.eu). A special thanks to Kate Burke for the English revision of the text.

\section{Appendix}

Table 1

\begin{tabular}{|c|c|c|c|}
\hline \multirow[b]{2}{*}{ Object } & \multicolumn{3}{|c|}{ Verbs } \\
\hline & Manipulation & Function & Observation \\
\hline Ball & Colpisci (to hit) & Gioca (to play) & $\begin{array}{l}\text { Osserva } \\
\text { (to look at) }\end{array}$ \\
\hline Bottle & Tappa (to plug up) & Versa (to pour) & $\begin{array}{l}\text { Guarda } \\
\text { (to look at) }\end{array}$ \\
\hline Brush & Stringi (to hold) & Pettina (to comb) & $\begin{array}{l}\text { Fissa } \\
\text { (to gaze) }\end{array}$ \\
\hline Controller & Appoggia (to support) & Premi (to push) & Vedi (to see) \\
\hline Fork & Raccogli (to pick up) & Mangia (to eat) & $\begin{array}{l}\text { Osserva } \\
\text { (to look at) }\end{array}$ \\
\hline Funnel & Prendi (to bring) & Travasa (to pour) & $\begin{array}{l}\text { Guarda } \\
\text { (to look at) }\end{array}$ \\
\hline Hammer & Impugna (to clasp) & $\begin{array}{l}\text { Batti } \\
\text { (to hammer) }\end{array}$ & $\begin{array}{l}\text { Fissa } \\
\text { (to gaze) }\end{array}$ \\
\hline Mug & Prendi (to bring) & Bevi (to drink) & Vedi (to see) \\
\hline Pan & Lava (to wash) & Cucina (to cook) & $\begin{array}{l}\text { Osserva } \\
\text { (to look at) }\end{array}$ \\
\hline Pen & Sposta (to move) & Scrivi (to write) & $\begin{array}{l}\text { Guarda } \\
\text { (to look at) }\end{array}$ \\
\hline Screwdriver & Posa (to put down) & Avvita (to screw) & $\begin{array}{l}\text { Fissa } \\
\text { (to gaze) }\end{array}$ \\
\hline Shovel & Afferra (to grasp) & Scava (to dig) & Vedi (to see) \\
\hline
\end{tabular}

\section{References}

Anelli, F., Nicoletti, R., \& Borghi, A. M. (2010). Categorization and action: What about object consistence? Acta Psychologia, 133, 203-211.

Binkofski, F., Dohle, C., Posse, S., Stephan, K. M., Hefter, H., Seitz, R. J., et al. (1998). Human anterior intraparietal area subserves prehension: A combined lesion and functional mri activation study. Neurology, 50, 1253-1259.

Borghi, A. M. (2004). Object concepts and action: Extracting affordances from objects parts. Acta Psychologica, 115, 69-96.

Borghi, A. M., \& Riggio, L. (2009). Sentence comprehension and simulation of object temporary, canonical and stable affordances. Brain Research, 1253, 117-128.

Boronat, C. B., Buxbaum, L. J., Coslett, H. B., Tang, K., Saffran, E. M., Kimberg, D. Y., et al. (2005). Distinctions between manipulation and function knowledge of objects: Evidence from functional magnetic resonance imaging. Brain Res Cogn Brain Res, 23(2-3), 361-373.

Bub, D. N., Masson, M. E., \& Cree, G. S. (2008). Evocation of functional and volumetric gestural knowledge by objects and words. Cognition, 106, 27-58.
Chemero, A. (2003). An outline of a theory of affordances. Ecological Psychology, 15, 181-195.

Chemero, A. (2009). Radical embodied cognitive science. Cambridge, MA: MIT Press.

Costantini, M., Ambrosini, E., Tieri, G., Sinigaglia, C., \& Committeri, G. (2010). Where does an object trigger an action? An investigation about affordances in space. Experimental Brain Research, 207, 95-103.

Costantini, M., \& Sinigaglia, C. (2011). Grasping affordance: A window onto social cognition. In A. Seemann (Ed.), Joint attention: New developments (p. XXX-XXX). Cambridge, MA: MIT press.

Creem, S. H., \& Proffitt, D. R. (2001). Grasping objects by their handles: A necessary interaction between cognition and action. Journal of Experimental Psychololgy: Human Perception and Performance, 27, 218-228.

Creem-Regehr, S. H., \& Lee, J. N. (2005). Neural representations of graspable objects: Are tools special? Brain Res Cogn Brain Res, 22, 457-469.

DeMauro, T., Mancini, F., Vedovelli, M., \& Voghera, M. (1993). Lessico di frequenza dell'italiano parlato. Milano, Italy: ETASLIBRI.

Ellis, R., \& Tucker, M. (2000). Micro-affordance: The potentiation of components of action by seen objects. British Journal of Psychology, 91, 451-471.

Galati, G., Committeri, G., Spitoni, G., Aprile, T., Di Russo, F., Pitzalis, S., et al. (2008). A selective representation of the meaning of actions in the auditory mirror system. NeuroImage, 40, 1274-1286.

Gallese, V. (2009). Motor abstraction: A neuroscientific account of how action goals and intentions are mapped and understood. Psychological Research, 73, 486-498.

Gerlach, C. (2009). Category-specificity in visual object recognition. Cognition, 111, 281-301.

Gibson, J. (1979). The ecological approach to visual perception. Boston, MA: Houghton-Mifflin.

Glenberg, A., \& Robertson, D. (2000). Symbol grounding and meaning: A comparison of high-dimensional and embodied theories of meaning. Journal of Memory and Language, 43, 379-401.

Jax, S. A., \& Buxbaum, L. J. (2010). Response interference between functional and structural actions linked to the same familiar object. Cognition, 115, 350-355.

Jeannerod, M. (2007). Being oneself. Journal of Physiology, 101, $161-168$

Kellenbach, M. L., Brett, M., \& Patterson, K. (2003). Actions speak louder than functions: The importance of manipulability and action in tool representation. Journal of Cognitive Neuroscience, $15,30-46$.

Mahon, B. Z., Schwarzbach, J., \& Caramazza, A. (2010). The representation of tools in left parietal cortex is independent of visual experience. Psychological Science, 21, 764-771.

Martin, A. (2007). The representation of object concepts in the brain. Annual Review of Psychology, 58, 25-45.

Menz, M. M., Blangero, A., Kunze, D., \& Binkofski, F. (2010). Got it! Understanding the concept of a tool. NeuroImage, 51, 14381444.

Murata, A., Fadiga, L., Fogassi, L., Gallese, V., Raos, V., \& Rizzolatti, G. (1997). Object representation in the ventral premotor cortex (area f5) of the monkey. Journal of Neurophysiology, 78, 22262230 .

Pellicano, A., Iani, C., Borghi, A. M., Rubichi, S., \& Nicoletti, R. (2010). Simon-like and functional affordance effects with tools: The effects of object perceptual discrimination and object action state. Quarterly Journal of Experimental Psychology, $63,1-12$ 
Rizzolatti, G., \& Matelli, M. (2003). Two different streams form the dorsal visual system: Anatomy and functions. Experimental Brain Research, 153, 146-157.

Scorolli, C., Borghi, A. M., \& Glenberg, A. (2009). Language-induced motor activity in bi-manual object lifting. Experimental Brain Research, 193, 43-53.
Tucker, M., \& Ellis, R. (1998). On the relations between seen objects and components of potential actions. Journal of Experimental Psychology: Human Perception and Performance, 24, 830-846.

Tucker, M., \& Ellis, R. (2001). The potentiation of grasp types during visual object categorization. Visual Cognition, 8, 769-800. 